\title{
Técnicas y características de los muros mixtos de tierra y madera. El caso de España
}

\section{Techniques and characteristics of traditional mixed walls using earth and timber. The case of Spain}

$\underline{\text { Francisco Javier Gómez-Patrocinio }}^{(*)}$, Lidia García-Soriano ${ }^{(* *)}$, Fernando Vegas ${ }^{(* * *)}$, Camilla Mileto ${ }^{(* * * *)}$

\section{RESUMEN}

Este artículo presenta un estudio de la construcción con muros mixtos de tierra y madera a través del análisis de 289 casos repartidos por toda España. Las variantes identificadas han sido agrupadas en familias constructivas con características comunes, con el objeto de facilitar su reconocimiento e interpretación. En el capítulo de discusión, se destaca qué tipos de subestructura de madera y de relleno de tierra son más frecuentes y se observa el modo en el que se suelen combinar entre ellos. En las conclusiones se reflexiona sobre cómo esta combinación encuentra un compromiso entre sentido de la oportunidad y adaptación al contexto - características fundamentales de las construcciones vernáculas - y da lugar a la enorme riqueza técnica de esta arquitectura. Este texto supone un importante avance en el conocimiento de los muros mixtos de tierra y madera, fundamental para poder plantear estudios orientados a la protección, rehabilitación y consolidación de los edificios erigidos con ellos, tanto en España como en otros países con soluciones constructivas similares.

Palabras clave: Arquitectura tradicional; Arquitectura de tierra; Entramados; Muros tejidos.

\section{ABSTRACT}

This article presents a study of the traditional construction with mixed walls using earth and timber based on 289 case studies throughout Spain. In order to facilitate their identification and interpretation, the variants in this study have been assorted into constructive groups with similar characteristics. In the discussion chapter, the most common kinds of timber structure and earthen infilling are highlighted, and the way in which they are usually combined is assessed. Conclusions reflect on how the combination of opportunity and adaptation to the context - characteristics essential to vernacular constructions - shed light on a wide range of alternative techniques in this architecture. This article further expands on the existing information on mixed walls using earth and timber, essential to propose studies and projects aiming to protect, retrofit and consolidate this type of architecture in Spain and other countries.

Keywords: Traditional architecture; Earthen architecture; Half-timber; Wattle-and-daub.

$\left(^{*}\right)$ Dr. Arquitecto. Técnico Superior de Investigación. Universitat Politècnica de València, Valencia (España).

$\left({ }^{* *}\right)$ Dra. Arquitecta. Profesora Asociada. Universitat Politècnica de València, Valencia (España).

$\left.{ }^{* * *}\right)$ Dr. Arquitecto. Catedrático de Universidad. Universitat Politècnica de València, Valencia (España).

$\left.{ }^{* * * *}\right)$ Dra. Arquitecta. Catedrática de Universidad. Universitat Politècnica de València, Valencia (España).

Persona de contacto/Corresponding author: fragmepa@upv.es (F. J. Gómez-Patrocinio)

ORCID: https://orcid.org/oooo-00o3-0560-2230 (F. J. Gómez-Patrocinio); https://orcid.org/oooo-0001-0315-6839 (L. García-Soriano); https://orcid.org/oooo-0003-0315-6839 (F. Vegas); https://orcid.org/oooo-0002-6987-8802 (C. Mileto)

Cómo citar este artículo/Citation: Gómez-Patrocinio, F. J.; García-Soriano, L.; Vegas, F.; Mileto, C.; (2021). Técnicas y características de los muros mixtos de tierra y madera. El caso de España. Informes de la Construcción, 73(561): e372. https://doi.org/10.3989/ic.73876

Copyright: (C) 2021 CSIC. Este es un artículo de acceso abierto distribuido bajo los términos de la licencia de uso y distribución Creative Commons Reconocimiento 4.0 Internacional (CC BY 4.0). 


\section{INTRODUCCIÓN}

La tierra es un material de construcción conocido desde la misma génesis de la Arquitectura y que ha sido ampliamente utilizado a lo largo de la historia. En España, este material cuenta con una especial importancia, tanto por la enorme cantidad de edificios construidos con él, como por su relevancia desde un punto de vista histórico y etnológico (1) (2).

En 1984, Houben y Guillaud identificaron doce formas de utilizar la tierra en construcción (3) y las agruparon en tres grandes grupos de sistemas constructivos: los muros monolíticos de tierra, los muros de albañilería de tierra y la tierra en estructuras mixtas. Los muros monolíticos son técnicas que se caracterizan por dar lugar a elementos unitarios, masivos y homogéneos que no generan juntas o lo hacen en número muy reducido. Pertenecen a este grupo técnicas como la tapia o la pared de mano (4). En los muros de albañilería de tierra se emplean piezas de dimensiones reducidas para construir elementos aparejados de fábrica. En la arquitectura tradicional española, estas piezas suelen aparecer en forma de adobes, tepes y terrones (5). Por último, la tierra en estructuras mixtas incluye tanto el uso de la tierra como revestimiento (pavimentos, cubiertas y enlucidos de tierra), como su empleo en la construcción de muros mixtos de tierra y madera.

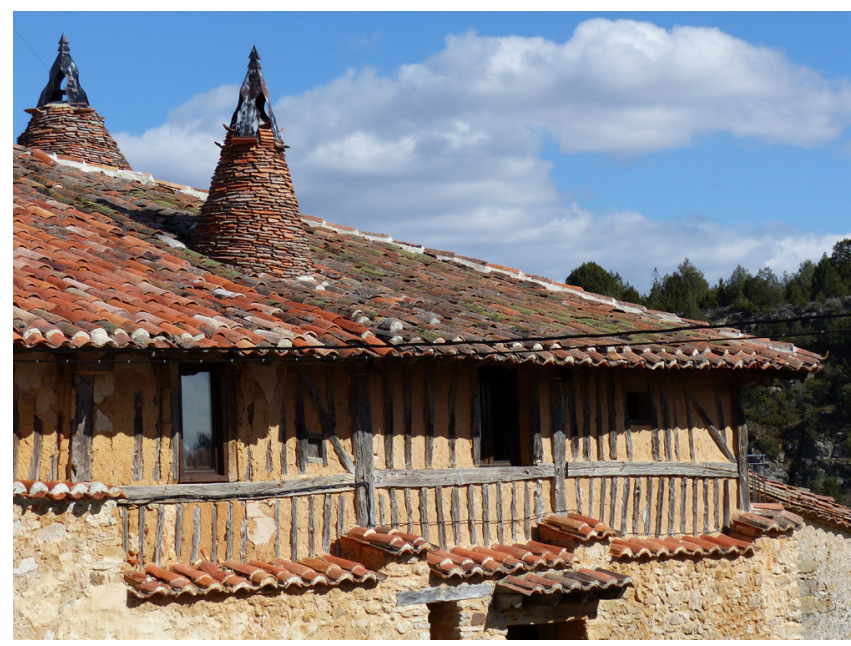

Figura 1. Construcción de entramado relleno de adobe en Calatañazor, Soria (F. Vegas y C. Mileto).

Los muros mixtos son sistemas constructivos complejos, propios de zonas con abundante de madera o donde la necesidad de aprovechar el suelo edificable hace necesario economizar el grueso de las paredes (6). Se caracterizan por una marcada especialización funcional y mecánica de los elementos que los componen y por un buen comportamiento frente a la acción de los terremotos (7) (8). Por el contrario, son más vulnerables al fuego y al ataque de insectos xilófagos que la mayoría de las técnicas de construcción con tierra (9). Son sistemas conocidos desde la Antigüedad y han sido identificados en sitios con una cultura sismo-resistente tan documentada como el yacimiento arqueológico de Caral en Perú, donde se han hallado restos de quincha construidos en torno al 3000 a.C. (10).

Los muros mixtos se suelen erigir sobre una planta baja de ladrillo o mampostería y pueden resolver por completo las envolventes de los pisos superiores, conformar planos confinados entre esquinas de piedra, o utilizarse como elementos de subdivisión interior. Se caracterizan por contar con una estructura isostática de elementos de madera dispuestos siguiendo un ritmo apretado, que en ocasiones recibe el nombre de imprenta (11). El material que completa los espacios definidos por la subestructura, denominados cuarteles (12), cumple una doble función: cierra el espacio interior y confina la subestructura de madera, que es la que asume las tareas portantes (Fig. 1). Sin embargo, en ocasiones los mecanismos de degradación que afectan a la madera, como la pudrición o los ataques de insectos xilófagos, empiezan a mermar su resistencia y los rellenos de tierra entran progresivamente en carga (13). Afortunadamente, estos procesos de deterioro son muy lentos y los elementos de tierra son solicitados poco a poco. Esto ralentiza la aparición de lesiones mecánicas y permite mantener la función estructural de los muros durante más tiempo.

Existe un sistema de construcción, denominado muro armado por algunos autores (11) (13), que surge de la combinación de un muro de adobe y un pórtico de madera y en el que la separación entre pies derechos es igual o superior a la altura entre forjados (Fig. 2). Con frecuencia, estas construcciones están ejecutadas con adobe en todas sus plantas - o cuentan únicamente con un zócalo de piedra o ladrillo - y en ellas las tareas estructurales se reparten entre los elementos de madera y los paramentos de tierra. Al no verificarse la especialización funcional que caracteriza a los entramados, los muros armados no se han incluido en este estudio.

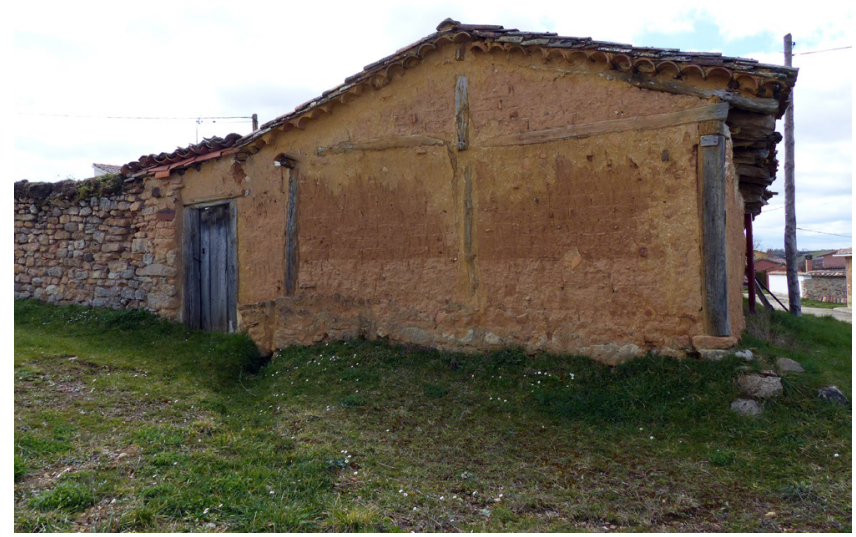

Figura 2. Construcción de adobe con pórticos de madera en Roscales de la Peña, Palencia (F. Vegas y C. Mileto).

Los muros mixtos de tierra y madera están vinculados habitualmente a tipologías edificatorias propias de entornos más bien urbanizados, y suelen contar con revestimientos destinados a proteger a los elementos que cierran los cuarteles y a dotarles de un acabado final. Con frecuencia, este revestimiento es un trullado de barro y paja similar al que se emplea para recubrir los muros de adobe. Sin embargo, dependiendo de los materiales disponibles en cada zona, de la importancia del edificio y de la capacidad económica de sus propietarios, el revestimiento puede no realizarse o llevarse a cabo con materiales más resistentes, como tejas, tablones, mortero de cal o pasta de yeso. Algunas variedades de muro mixto realizadas con encofrados, como los entramados rellenos con tapialete, permiten obtener un acabado durante la construcción del muro que hace innecesario el empleo de revestimientos. 


\subsection{Muros entramados y muros tejidos}

Dependiendo del modo en el que la subestructura de madera se relaciona con los elementos que cubren sus cuarteles, es posible distinguir entre dos tipos de muros mixtos: los entramados o muros de telar y los muros tejidos (14).

En los entramados, las subestructuras de madera se combinan con un relleno - o forjado - de otro material, que completa la pared y que se encuentra poco trabado con la imprenta que lo sostiene. Estos rellenos suelen estar constituidos por materiales pesados y con una cierta capacidad resistente, como fábrica de ladrillo o adobe, mampostería o rellenos vertidos de piedra que son mezclados normalmente con yeso, pues se trata de un material particularmente compatible con la madera (15). Esto les permite confinar la imprenta para evitar deformaciones en el plano de la fachada y asumir parte de las labores de la estructura cuando ésta empieza a degradarse (13). Sin embargo, los rellenos de los entramados se suelen encontrar pobremente trabados con las barras que sostienen el muro y pueden desprenderse ante deformaciones moderadas que saquen al muro de su plano. De acuerdo con el tratado de Ger y Lóbez, es posible recurrir a diferentes estrategias para mejorar la traba entre la imprenta y el relleno: proporcionar aspereza a la madera o envolverla en tomizas de esparto; formar cajas o ranuras en los pies derechos, clavando listones en los cantos de los postes o practicándoles escopladuras, para que entre el material de relleno; o colocar clavos en los pies derechos a modo de conectores, aunque con el tiempo estas piezas pueden desprenderse y perder su función. Durante la ejecución de los entramados, resulta conveniente colocar por el exterior del cuartel que se vaya a macizar un tablero que lo cierre y sostenga los ladrillos, adobes o cascotes del relleno, aun cuando éstos no vayan a ser vertidos (12). Una vez macizada una porción, este tablero se traslada al siguiente cuartel, que se resarce del mismo modo.

Por su parte, en los muros tejidos la subestructura de madera se completa con fibras o urdimbres vegetales que se entrelazan a los pies derechos para generar cerramientos ligeros y bien trabados. Estos elementos no tienen capacidad para asistir estructuralmente a la imprenta cuando ésta empieza a degradarse, pero son más ligeros e introducen menos solicitaciones sobre los muros inferiores. Además, no presentan los problemas de adherencia que en ocasiones afectan a los entramados. Sobre las urdimbres de fibras se suele aplicar un recubrimiento grueso de barro, muchas veces extendido directamente con la mano, que sirve para incrementar la consistencia y durabilidad de los tabiques, proteger a las fibras frente a la pudrición y dotar a los cerramientos de estanqueidad al aire y a la luz.

\section{ESTADO DEL ARTE}

Las crisis energéticas de los años 70 (16) provocaron la aparición en Europa de un interés creciente por el estudio de la arquitectura de tierra. Esta nueva atención vino motivada por diferentes factores, entre los que cabe destacar los siguientes: la voluntad de desarrollar una arquitectura contemporánea más sostenible y compatible con un planeta con recursos limitados; la existencia de un abundante patrimonio histórico arquitectónico construido con tierra que debe ser atendido; o la necesidad de dar alternativas de construcción económica y segura a las personas que habitan en casas de tierra y que actualmente suponen más de una cuarta parte de la pobla- ción mundial (17). Con la intención de dar respuesta a estas inquietudes, a partir de los años setenta se organizaron los primeros congresos internacionales sobre arquitectura, como las International Conferences on the Conservation of Mudbrick Monuments (Yadtz, 1972 y 1976; Ankara, 1980; etc.), y aparecieron los primeros libros y manuales de construcción con tierra (entre otros: (3) (18)).

A partir de los años 80, el contexto internacional propició la aparición en España de un interés por la restauración del patrimonio monumental construido con tierra, que fue creciendo a lo largo de las siguientes décadas (entre otros: (19) (20)) y que ha llegado a cristalizar en el desarrollo de estudios en profundidad y trabajos panorámicos sobre la restauración de edificios monumentales construidos en tapia (entre otros: (21) (22)). Sin embargo, ha sido necesario esperar algunas décadas más para que despierte una verdadera voluntad por el estudio de la arquitectura vernácula construida con tierra. Prueba de ello es que, tras la publicación de los últimos tratados de construcción previos a la definitiva implantación del cemento en la construcción (entre otros: (12) (15) (23)), estas técnicas cayeron prácticamente en el olvido y no resurgió un interés real por su recuperación hasta prácticamente el cambio de milenio. En el caso de los muros mixtos, esta nueva sensibilidad ha surgido de la mano de estudios centrados en las técnicas existentes en regiones concretas del país (9) (24) (25), en la etimología de los términos constructivos (11) o en el funcionamiento de la subestructura (26). Sin embargo, no existen trabajos que aborden la variedad de soluciones de colmatación de los cuarteles de una forma global. El presente artículo trata de cubrir esta ausencia y examina los principales resultados obtenidos en un análisis panorámico de la arquitectura tradicional española construida con muros mixtos de tierra y madera.

\section{OBJETIVOS}

El conocimiento es un requisito esencial para la conservación. Sólo aquello que tiene un nombre puede ser mencionado y, como consecuencia, reconocido y valorado. Por esta razón, el principal objetivo del presente artículo es identificar y comprender las principales técnicas y variantes de construcción con muros mixtos de tierra y madera que existen en España. Este conocimiento y esta identificación pueden contribuir sin duda a la valoración de esta arquitectura y favorecer su preservación.

Como punto de partida, este artículo pretende ofrecer un panorama global de las técnicas de construcción con muros mixtos de tierra y madera utilizadas en la arquitectura tradicional española. Para conseguirlo, comprende la caracterización constructiva de una amplia muestra de casos de estudio repartidos por todo el país. A partir de este objetivo general, es posible señalar una serie de objetivos específicos:

- Identificar las principales técnicas de construcción de muros mixtos de tierra y madera existentes en España, describiendo sus principales características y estudiando su proceso constructivo.

- Reconocer las diferentes variantes de estas técnicas, detallando sus particularidades y características.

- Clasificar las técnicas y variantes registradas para facilitar su comprensión y asistir en el análisis constructivo de nuevos casos de estudio.

- Reflexionar sobre las características de los muros mixtos de tierra y madera en España, para fomentar una mejor 
comprensión de esta arquitectura, favorecer su percepción como un conjunto patrimonial valioso que debe ser protegido y proporcionar una base de conocimiento que permita una intervención compatible.
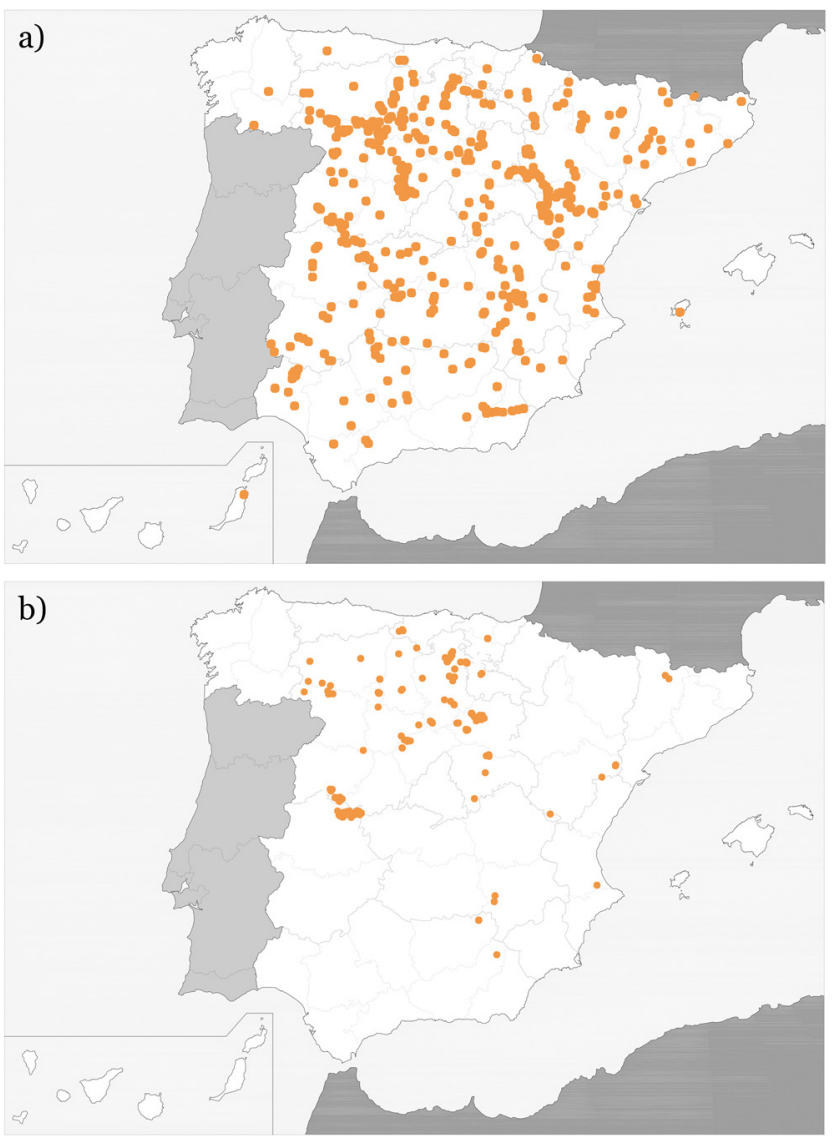

Figura 3. Ubicación de los casos de estudio recogidos en la base de datos completa (a) y de los casos de construcción de los muros mixtos que han conformado la muestra (F. J. Gómez-Patrocinio).

\section{METODOLOGÍA}

La metodología empleada en el trabajo presentado en este artículo se ha basado en el análisis de casos de estudios extraídos de una extensa base de datos de arquitectura tradicional de tierra en España. Durante la formación de dicha base de datos, ha sido posible documentar 1.696 construcciones de tierra repartidas en 328 municipios de 40 provincias españolas (Fig. 3a). Los casos contenidos en esta base de datos incluían ejemplos de las diferentes técnicas de construcción con tierra presentes en el país, como la tapia, la albañilería de adobe o los muros mixtos de tierra y madera. La investigación presentada en el artículo abarca los casos construidos con muros mixtos y ha implicado el análisis de 289 construcciones distribuidas entre 90 municipios de 24 provincias (Fig. 3b) (27).

La muestra resultante ha sido analizada aplicando una serie de procedimientos cualitativos y cuantitativos. El análisis cualitativo ha implicado el reconocimiento in situ de los edificios, el análisis detallado de su sistema constructivo y el mapeado geográfico de los casos. Por su parte, los procedimientos cuantitativos han conllevado el estudio estadístico de la presencia de las diferentes variantes con respecto a la muestra total. Esta metodología aspiraba a identificar las diferentes técnicas de construcción con muros mixtos de tierra y madera utilizadas en la arquitectura tradicional española y definir sus características principales, su ámbito de aparición y la frecuencia de su uso.

La caracterización constructiva de los casos de estudio se ha acometido a través de un análisis taxonómico basado en una serie de clasificaciones sucesivas. Este método permite atender por separado a diferentes aspectos de la técnica, dando una descripción rápida y completa de cada ejemplo a partir de la combinación de los diferentes apartados. Durante su caracterización constructiva, cada uno de los edificios estudiados se ha clasificado atendiendo a diferentes factores:

- Geometría de la imprenta de madera: Describe la configuración de la subestructura de madera que sostiene el muro, en función de si está formada por elementos verticales, horizontales, diagonales o por una combinación de ellos (Fig. 4a).

- Familia de muros mixtos: Indica si la variante estudiada se corresponde con la de un entramado, donde los huecos de la subestructura son resarcidos con un relleno de tierra, o con un muro tejido, donde la subestructura de madera se completa con elementos de tierra y fibras que se entrelazan con ella.

- Tipo de cerramiento: Especifica las características particulares del material que rellena o teje el espacio entre los elementos de la subestructura de madera (Fig. 4b).

- Presencia de revestimientos: En este apartado se estudia la presencia de revestimientos de cualquier índole, generalmente de barro y paja, madera, teja cerámica, mortero de cal, pasta de yeso o una combinación de ellos (Fig. 4c).

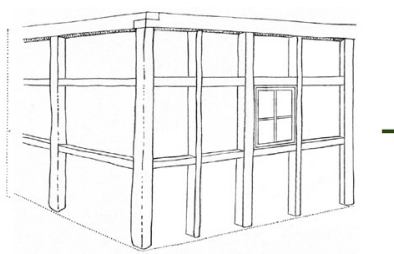

a)

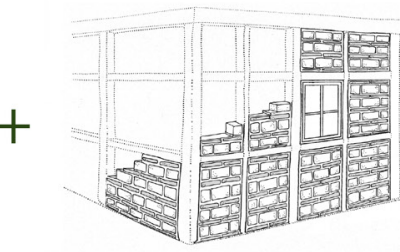

b)

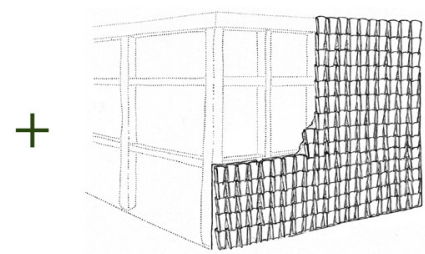

c)

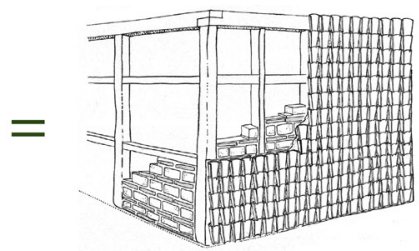

Total

Figura 4. Sistema de clasificaciones sucesivas para la caracterización constructiva de los casos de estudio (F. J. Gómez-Patrocinio). 
La información relativa a las técnicas identificadas se ha registrado en una base de datos mediante el empleo de una ficha específica. Los métodos de estudio sistematizados mediante el empleo de fichas son herramientas que facilitan el análisis constructivo de los casos, asistiendo en el proceso de toma de datos y facilitando la comparación entre edificios y la extracción de conclusiones (28) (29). Las fichas empleadas en este estudio presentan la siguiente estructura (Fig. 5):

- Descripción general de la técnica: título descriptivo, código en función de la nomenclatura preestablecida, filiación de la técnica dentro de una familia constructiva y tabla de descripción para una rápida identificación de sus características.

- Datos generales de la técnica: número de casos identificados, tipología mayoritaria, tipo de uso principal, emplazamiento de los casos y un caso principal representativo de la técnica descrita.

- Caracterización constructiva y estructural: Geometría de la imprenta, Familia de muros mixtos, Tipo de muro mixto y presencia de Revestimientos.

- Observaciones.

- Otros casos, donde se complementa el caso principal con hasta tres ejemplos más.

El sistema de clasificación empleado permite una fácil reestructuración de la información para organizar los ejemplos en diferentes familias. Gracias a ello, posibilita la obtención de resultados que atiendan a clasificaciones parciales según el aspecto a considerar. Los resultados aportados por este sistema de catalogación son tanto cualitativos como cuantitativos pues, al ser aplicado sobre una muestra grande, permiten determinar la representatividad de cada variante dentro de esta arquitectura. Los resultados del estudio se han agrupado en familias de técnicas con una lógica constructiva similar. Las diferentes técnicas identificadas dentro de cada familia se han distinguido como variantes comunes o como variaciones aisladas, dependiendo de si su uso es habitual en la muestra o si su aparición es anecdótica (27).

La presencia mínima a partir de la cual una variación podía ser considerada variante común se ha establecido en un $5,00 \%$ de los casos registrados en la familia. La siguiente ecuación [1] ha sido utilizada para estimar la fiabilidad de la muestra y sus estratos, y para obtener el error muestral previsible (30):

$$
e=\sqrt{ }\left(\left(z^{2} \cdot p \cdot q\right) / n\right)
$$

Desde un punto de vista estadístico, los ejemplos estudiados se han considerado como $\mathrm{n}$ individuos de una población infinita $(\mathrm{N}>30.000)$. El nivel de confianza en los resultados se ha establecido en un valor estándar del 95\% ( $\alpha$ $=5 \%$ ), traducido en un valor $z$ de 1,96 . La variabilidad esperada de las respuestas de la población se ha fijado en un valor máximo ( $p=q=o, 5)$. El error muestral considerado en el estudio queda establecido de esta manera en un $5,8 \%$.

\section{RESULTADOS}

\subsection{Aproximación a la geometría de las imprentas de madera}

Los muros mixtos se caracterizan por contar con una densa subestructura de madera en la que aparecen pies derechos de

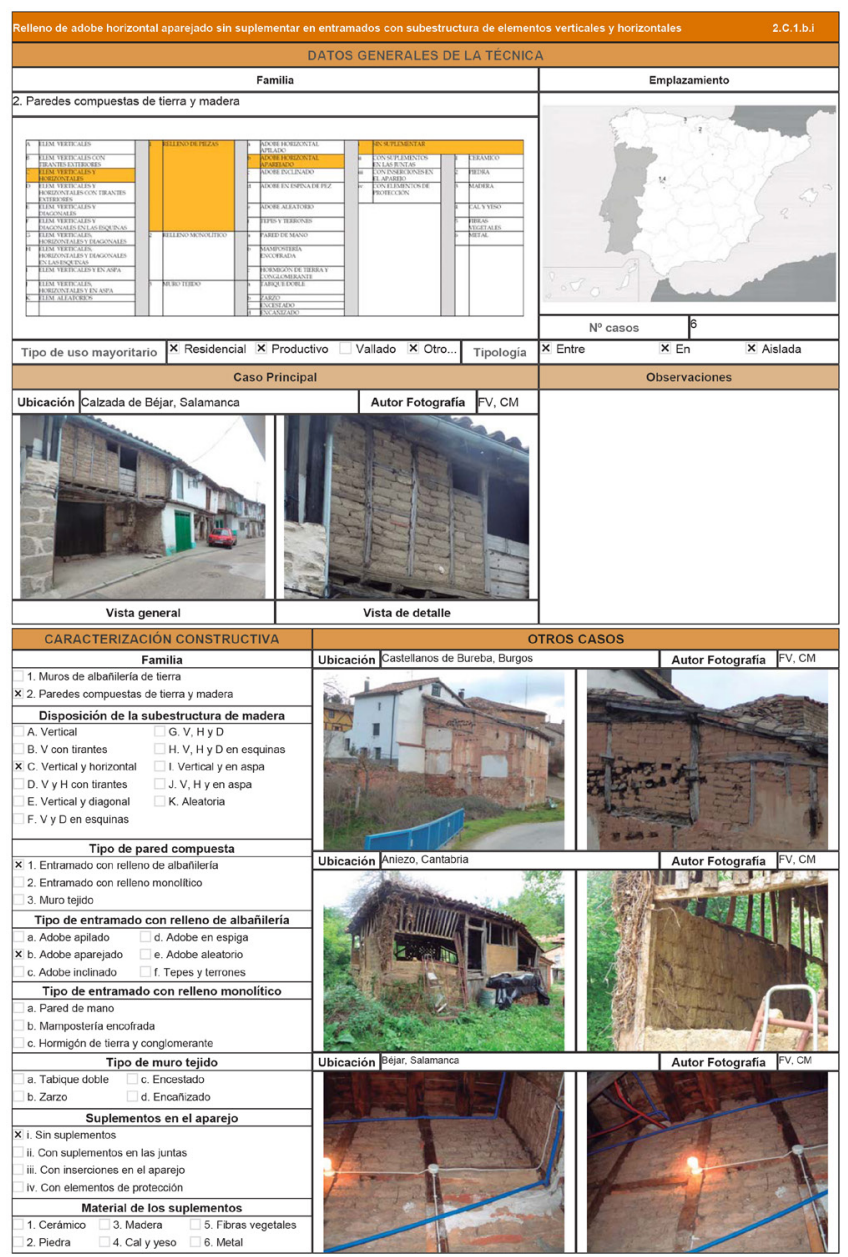

Figura 5. Ficha empleada para registrar los resultados de la caracterización constructiva (F. J. Gómez-Patrocinio).

poco espesor siguiendo ritmos apretados, muchas veces con separaciones inferiores a 1,5 metros. Las distintas barras que componen estos cerramientos han recibido tradicionalmente nombres diferentes, dependiendo de su posición (a partir de (6), (12) y (15)) (Fig. 6).

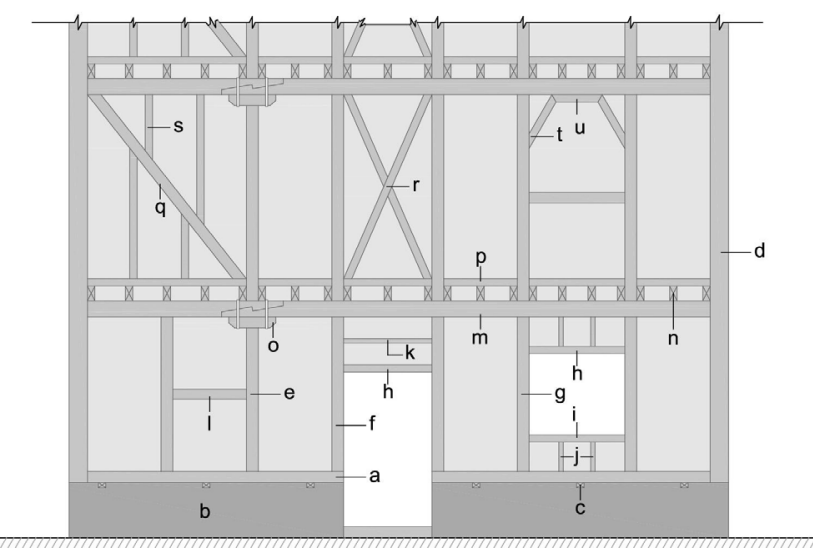

Figura 6. Denominación de los elementos que componen la imprenta de madera, en función de su emplazamiento (F. J. Gómez-Patrocinio).

Estos muros suelen arrancar de soleras de madera (a) fijadas a una planta baja, zócalo o citarón de ladrillo o mampostería (b) mediante pequeñas piezas de madera - nudillos (c) - empotradas en él. Sobre las soleras apoyan los elementos verti- 
cales que conforman la base de la trama. Estos pies derechos se denominan postes de ángulo o cornijales (d) si resuelven una esquina del muro, almas si resuelven la intersección entre dos paños y postes de relleno (e) si se encuentran embebidos en el interior del paño. Cuando definen el hueco de una puerta, reciben el nombre de postes de cerco o postes de lección (f) y cuando delimitan una ventana se llaman largueros (g). En estos casos, cuentan con piezas horizontales que cierran el vano por arriba y por abajo, recibiendo el nombre de cabeceros (h) y peanas (i) respectivamente. El espacio entre estas piezas y las soleras o carreras se suele dividir mediante pilarejos (j) o contrapuentes (k). Cuando el paño definido por dos pies derechos es excesivamente esbelto, puede ser dividido en dos cuarteles más pequeños por un madero horizontal denominado puente (l).

En su extremo superior, los pies derechos de cada piso sostienen una carrera $(\mathrm{m})$ sobre la que descansarán las viguetas (n) del forjado superior o los pares de la cubierta. Debido a su longitud, las carreras y las soleras suelen estar compuestas por varias piezas ensambladas. En estos casos, los empalmes se deben realizar sobre los pies derechos y muchas veces se resuelven empleando zapatas (o) y abrazaderas de hierro para mejorar el apoyo. Los pies derechos del nivel superior descansan habitualmente sobre la carrera del forjado situado bajo ellos. En ocasiones, estos postes también pueden arrancar de la sobrecarrera (p) del piso superior, resultando en una fijación de los postes algo más débil. El empleo de tornapuntas o riostras (q) o de cruces de San Andrés (r) mejora el atado de los muros, al dividirlos en triángulos indeformables y dotarles de una mayor rigidez. Cuando los cuarteles definidos por postes y tornapuntas son excesivamente amplios, es posible reforzar la estructura empleando virotillos (s), jabalcones $(\mathrm{t})$ y sopandas $(\mathrm{u})$.

En los cerramientos más simples, la disposición próxima de los postes de relleno permite la formación de cuarteles verticales y estrechos que discurren a lo largo de toda la altura entre forjados. Sin embargo, en los entramados es frecuente encontrar estos pies derechos combinados con tornapuntas y cabeceros para dar lugar a imprentas más complejas. En los muros tejidos también es posible encontrar tornapuntas y otros elementos de refuerzo de la estructura, pero normalmente se trata de barras aisladas y su aparición es mucho más esporádica.

Las combinaciones de elementos verticales, horizontales y diagonales que es posible encontrar son múltiples y permiten obtener imprentas más rígidas y resistentes, ya que interconectan los pies derechos, reduciendo su longitud de pandeo e incrementando la capacidad del muro para absorber esfuerzos horizontales. Existe una tipología de muros entramados particularmente compleja, que algunos autores han denominado entramados estrellados (11), que se caracteriza por la formación de paños rectangulares que son divididos por cruces de San Andrés o tornapuntas dispuestos en aspa, dando lugar a pequeños cuarteles triangulares.

En ocasiones, también es posible encontrar muros mixtos en los que los pies derechos han sido conectados entre sí mediante elementos horizontales o diagonales clavados a ellos por el exterior del cerramiento, sin romper la continuidad de los cuarteles. Probablemente, estas barras se hayan instalado durante la vida útil del edificio a fin de mejorar su rendimiento estructural. Sin embargo, su funcionamiento depende de la resistencia a la cizalladura de los clavos que las fijan, por lo que es probable que cuenten con una efectividad limitada.

\subsection{Técnicas de construcción de muros mixtos de tierra y madera en España}

El método de clasificación empleado ha permitido la identificación de 9 soluciones habituales de construcción con muros mixtos de tierra y madera, así como 7 alternativas poco frecuentes. Con el objeto de facilitar su identificación, estas 16 variantes han sido agrupadas en las 3 familias que se presentan en esta sección (Tabla 1). De igual modo, se incluye un subapartado en el que se recogen las soluciones de revestimiento que más habitualmente han sido empleadas para su protección.

Tabla 1. Presencia estadística de cada variante constructiva dentro de su familia y de la muestra completa.

\begin{tabular}{|c|c|c|}
\hline Variante constructiva & \% Familia & \% Total \\
\hline Entramados con albañilería & $100 ’ 00 \%$ & $75^{\prime} 26 \%$ \\
\hline Rellenos de adobe apilado & $40 ’ 74 \%$ & $30 ’ 66 \%$ \\
\hline Rellenos de adobe aparejado & $36{ }^{\prime} 57 \%$ & $2753 \%$ \\
\hline Rellenos de adobe inclinado & 3'70\% & 2'79\% \\
\hline Rellenos de adobe en espiga & 2'78\% & 2’o9\% \\
\hline Rellenos de adobe aleatorio & $13 ’ 43 \%$ & $10 ’ 10 \%$ \\
\hline Rellenos de adobe y ladrillo & o’46\% & o’35\% \\
\hline Rellenos de tepes o terrones & 2’31\% & $1 ' 74 \%$ \\
\hline Entram. con relleno monolítico & 100'oo\% & $14 ' 63 \%$ \\
\hline Rellenos de pared de pellas & $2,38 \%$ & o’35\% \\
\hline Rellenos de pared de montón & $2,38 \%$ & o’35\% \\
\hline Rellenos de tierra y conglomerante & $42,86 \%$ & 6’27\% \\
\hline Rellenos de mampostería encofrada & $52 ’ 38 \%$ & 7'67\% \\
\hline Muros tejidos & 100'0o\% & 10'10\% \\
\hline Paredes entrelazadas & $65,52 \%$ & 6’62\% \\
\hline Mimbres & $6,90 \%$ & o’70\% \\
\hline Cañizos & $10,34 \%$ & 1'05\% \\
\hline Tabiques de tablas & $3,45 \%$ & o’35\% \\
\hline Emparrillados & $13 ’ 79 \%$ & 1'39\% \\
\hline
\end{tabular}

\subsubsection{Muros entramados con rellenos de albañilería de tierra}

En los entramados de esta familia, los cuarteles definidos por la imprenta de madera aparecen colmatados con adobe o, esporádicamente, con terrones o tepes. Las piezas que constituyen estos rellenos se colocan habitualmente a soga o a panderete, aunque puntualmente es posible encontrar otras soluciones. El empleo de estos aparejos, de poco espesor, permite reducir el material consumido en la plementería, cuya única función estructural es el arriostramiento de la trama lignaria, aligerando el muro y aumentando la superficie útil de la construcción.

Dependiendo del nivel de traba entre las piezas, es posible distinguir entre dos lógicas de colmatación de los cuarteles con adobes colocados en horizontal. En los rellenos de adobe horizontal aparejado, los bloques se disponen realizando un esfuerzo consciente por evitar la aparición de juntas continuas, cortando piezas para ello cuando es necesario (Figs. 
7 a y 7 b). Cuando este tipo de relleno se emplea para colmatar un entramado que cuenta con tornapuntas, las piezas que se disponen en el cuartel situado sobre la diagonal mantienen la horizontalidad y las hiladas van ganando longitud para ajustarse al ancho creciente del espacio a rellenar. Una variante similar son los rellenos de adobe horizontal apilado. Este tipo de plementería se emplea habitualmente para rellenar entramados cuyos pies derechos se encuentran muy próximos y pueden ser colmatados empleando una única pieza a soga o a panderete (Fig. 7c). El aparejo a panderete es común cuando el entramado constituye un tabique de partición interior sin función de aislamiento (Fig. 7d). En los casos en los que una pieza no es suficiente para completar el ancho del cuartel, la lógica de apilamiento prescinde de cualquier consideración sobre la interrupción de las juntas verticales, dando lugar a rellenos formados por columnillas adyacentes de adobe (Fig. 7e).

La disposición inclinada de las piezas permite absorber las irregularidades de los cuarteles y hacer menos evidentes las diferentes distancias entre montantes. Al emplear estos aparejos, es posible alterar libremente el ángulo de inclinación de las piezas, ajustándose al espacio disponible y rellenando los huecos entre los adobes y la imprenta con mortero de barro. De este modo, resulta más sencillo colmatar el cuartel con piezas enteras. Además, la colocación de las piezas en diagonal genera un cierto apoyo de éstas sobre los postes que flanquean el cuartel, incrementando el confinamiento de los pies derechos y reduciendo el riesgo de caída del relleno. De- pendiendo de si las piezas inclinadas se colocan en una única dirección o si se disponen en dos, será posible distinguir entre rellenos de adobe en diagonal (Fig. 7f) y rellenos de adobe en espiga o espina de pez (Fig. $7 \mathrm{~g}$ ).

A pesar de sus diferentes características, las cuatro variantes descritas anteriormente presentan una intencionalidad en la forma en la que se colocan los adobes. Sin embargo, es posible encontrar entramados en los que la disposición de las piezas se va modificando para ajustarse a cada cuartel y completarlo sin tener que hacer cortes. Estos rellenos de adobe dispuesto aleatoriamente dan lugar a plementerías de aspecto desordenado que habitualmente se regularizan mediante un revestimiento. Cuando este tipo de relleno se emplea para colmatar imprentas con tornapuntas, el cuartel situado debajo de la diagonal se suele rellenar empleando piezas aparejadas horizontalmente que descansan sobre la solera inferior. Por su parte, el cuartel superior se colmata habitualmente con un aparejo inclinado en paralelo a la diagonal o con piezas dispuestas en abanico que van absorbiendo el ángulo existente entre las tornapuntas y la carrera superior.

A pesar de que resultan poco comunes, durante el desarrollo de la investigación recogida en este texto se ha identificado la existencia de entramados rellenos con terrones, además del empleo de tepes para cerrar hastiales sostenidos por una subestructura de madera con unas características similares a las de un pequeño tramo de entramado. Estas piezas, que se

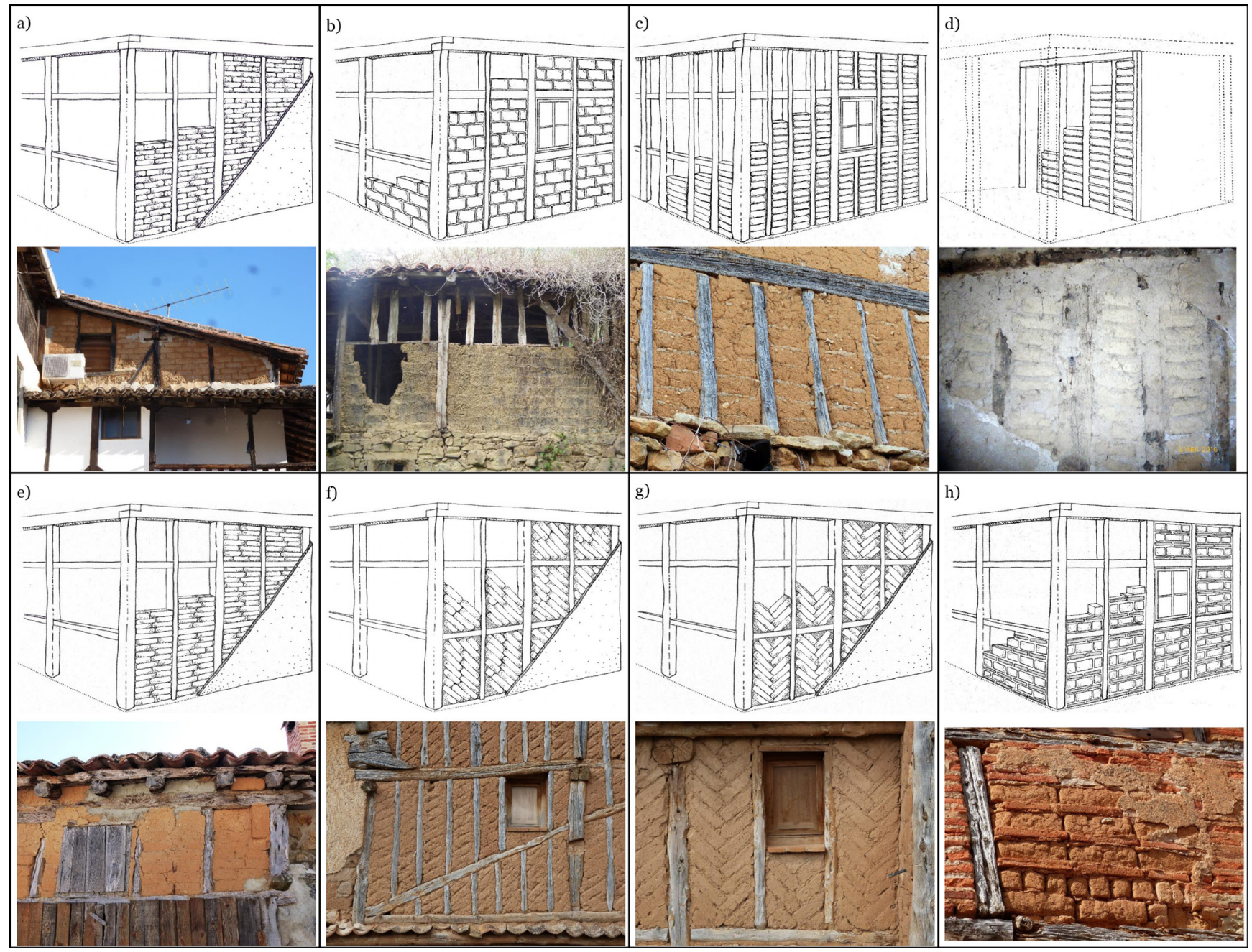

Figura 7. Entramados rellenos de albañilería de tierra (Dibujos: SOStierra. Fotografías: F. Vegas y C. Mileto). 
cortan directamente del terreno y se ponen en obra sin haber sido transformadas, han sido colocadas horizontalmente en todos los casos registrados y en su disposición es posible reconocer un esfuerzo por evitar la aparición de juntas continuas. De igual modo, se ha documentado el uso puntual de adobes y ladrillos para rellenar entramados formando aparejos con hiladas alternas (Fig $7 \mathrm{~h}$ ).

En la mayor parte de los casos, las juntas de las fábricas están constituidas por un mortero de barro y paja, o de barro y cal, de composición similar a la de los propios adobes. Sin embargo, también es común el uso de morteros de cal o yeso para la fijación de las piezas. A pesar de que los entramados se suelen rematar con un revestimiento, sólo ha sido posible identificar un caso en el que se han complementado las juntas con inserciones cerámicas para mejorar la conexión entre el muro y su acabado.

\subsubsection{Muros entramados con rellenos monolíticos}

Esta familia está conformada por técnicas que dan lugar a rellenos con un carácter unitario, masivo y homogéneo. El material empleado en su construcción puede colocarse apilado o vertido. Habitualmente, se ejecutan utilizando un encofrado por la cara exterior del entramado. Sin embargo, éste puede no utilizarse o ser un encofrado a dos caras.

Los rellenos apilados son soluciones poco comunes que se conforman por amontonamiento del material y que suelen presentar una compacidad y una resistencia mecánica reducidas. La pared de pellas es una solución que se construye por superposición de porciones de tierra unida, prieta y modelada hasta adquirir una forma redondeada (Fig. 8a). Se trata de una solución que resulta en la construcción de un cerramiento de pared de mano en forma de glebas frescas, que le dotan de un aspecto característico y fácilmente identificable. El empleo de un encofrado por la cara exterior del entramado permite asegurar la planeidad del muro y aporta una cierta compactación a la superficie externa del relleno. Por su parte, la pared de montón se basa en la simple acumulación de tierra que no recibe ningún tipo de modelado previo a su puesta en obra. El empleo de estas variantes sólo ha sido identificado en casos situados en Cantabrana (Burgos) y Calatañazor (Soria), respectivamente.
Los rellenos vertidos suelen combinar la tierra con materiales conglomerantes y con otros áridos, como grava o incluso mampuestos. Estos sistemas dan lugar a cerramientos pesados y resistentes cuya elevada consistencia se debe a la acción combinada de la capacidad aglomerante de la arcilla y al fraguado de la cal o el yeso. Las técnicas vertidas se basan en el empleo de mezclas bastante fluidas que adquieren estabilidad después de su puesta en obra, y en cuya construcción se hace necesario el empleo de encofrados. El tipo de relleno vertido más común es el tapialete (Fig. 8b). Su empleo es muy frecuente en las provincias de Teruel y Guadalajara, aunque también está presente en algunas zonas de Cuenca, Soria, Burgos y La Rioja, además de en algunas comarcas interiores de Valencia y Castellón (14). Está constituido por una mezcla de yeso y mampuestos que, gracias a su rápido fraguado, puede ser puesta en obra utilizando únicamente un encofrado exterior.

Diversos tratados (6) (15) recomiendan de forma expresa el empleo del yeso como material conglomerante en las obras entramadas, debido a su buena compatibilidad química con la madera. Sin embargo, algunos de los casos de estudio recogidos en este trabajo parecen estar colmatados con una mezcla de tierra, cal y grava mucho más semejante a un hormigón de cal y tierra (Fig. 8c). Diferentes estudios realizados en mezclas similares, empleadas en la construcción de muros de tapia, afirman que estos materiales suelen contar con proporciones de cal superiores al 20\% (21). Sin embargo, en mezclas con un contenido mucho menor, incluso inferior al 10\%, ésta ya adquiere un rol fundamental en la estabilización de la masa de tierra (31). De la adición de una cantidad considerable de mampuestos a una mezcla de tierra, grava y cal, similar a la descrita anteriormente, surgen los rellenos de mampostería encofrada (Fig. 8d).

\subsubsection{Muros tejidos}

Las variantes incluidas en esta familia se caracterizan por el empleo de diferentes elementos de origen vegetal para conformar pantallas ligeras que se fijan o entrelazan con la subestructura de madera para formar un cerramiento.

Probablemente, los elementos que mejor ejemplifican el concepto de muro tejido son las paredes entrelazadas (Fig. 9a), como los zarzos, los setos o los verganazos, presentes en la arquitectura tradicional de provincias como León, Palencia,

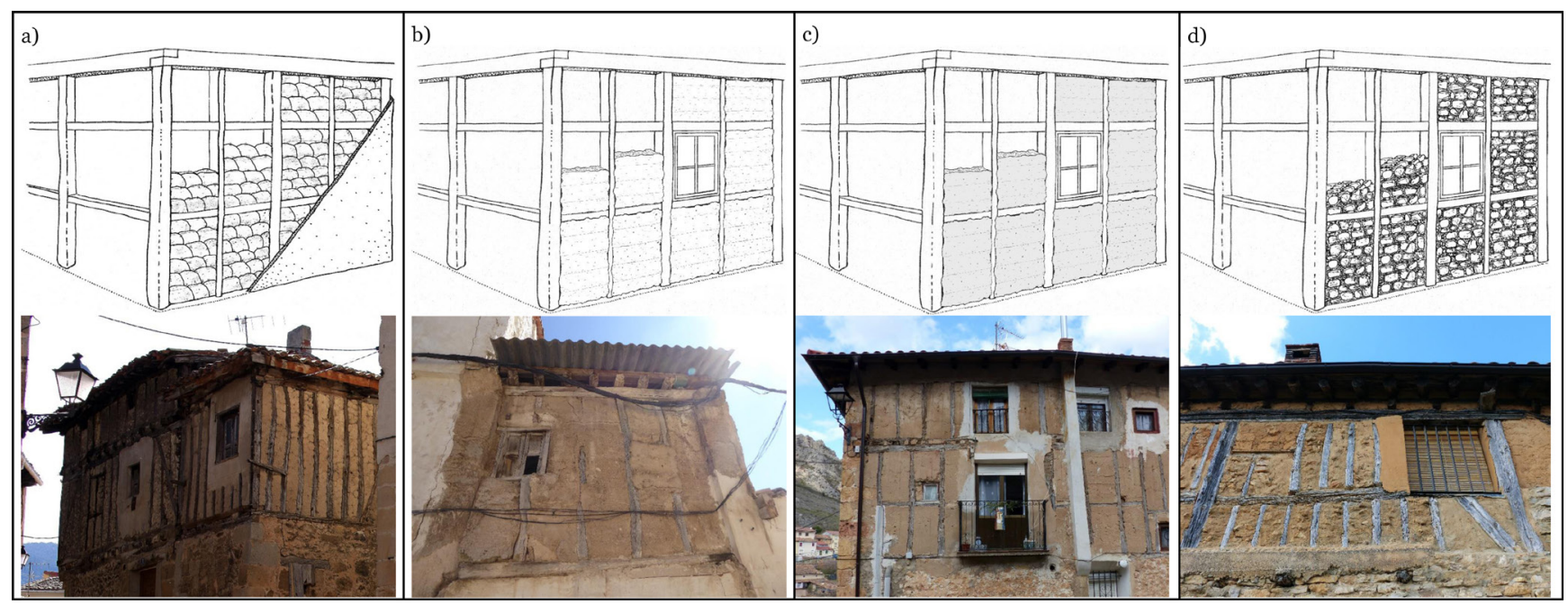

Figura 8. Entramados con rellenos monolíticos (Dibujos: SOStierra. Fotografías: B. Arnáiz (a), F. Vegas y C. Mileto (b, c, d)). 


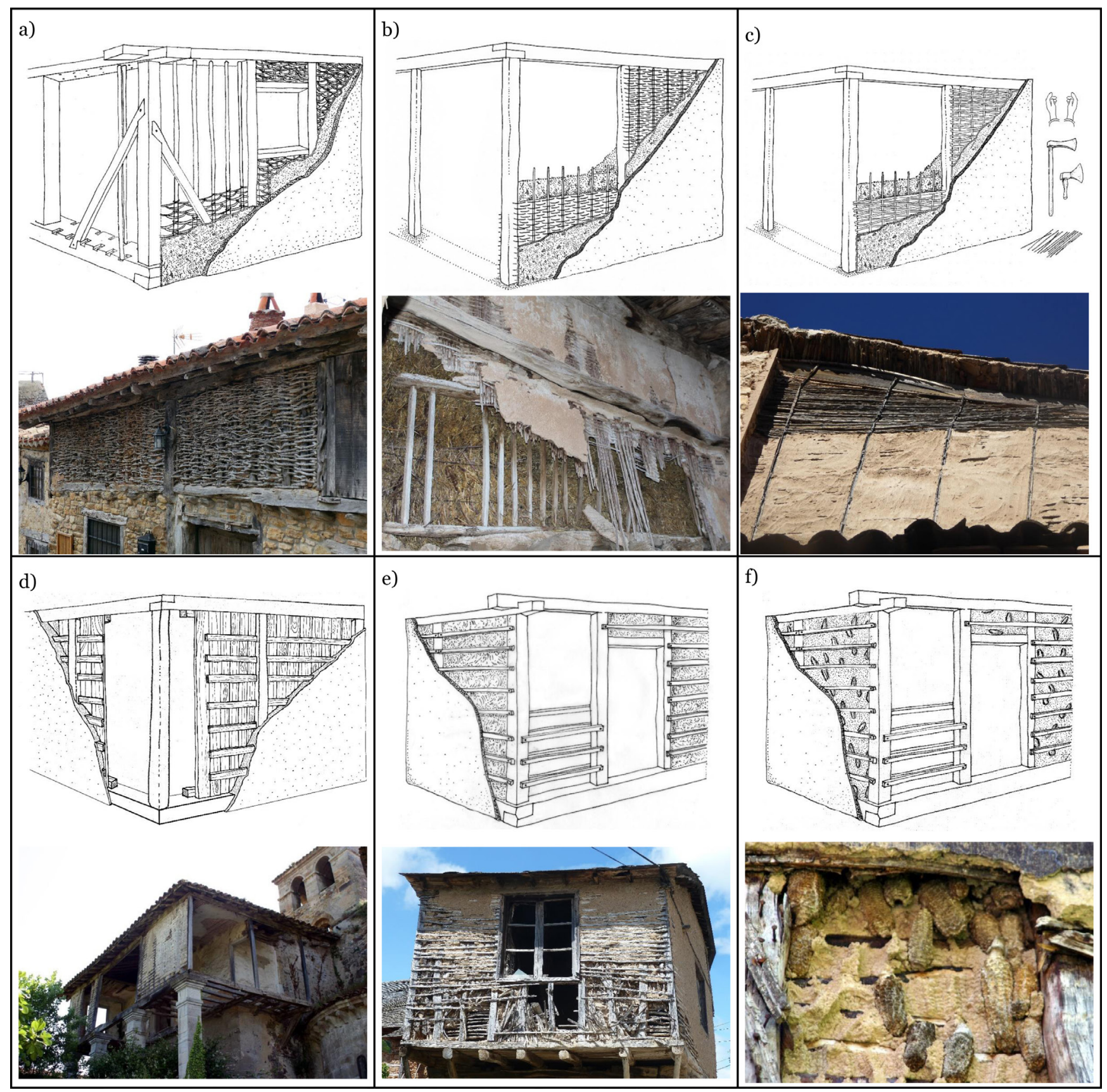

Figura 9. Muros tejidos (Dibujos: SOStierra. Fotografías: S. Tomás (b), J. Font (d, e), F. Vegas y C. Mileto (a, c, d)).

Soria o Cantabria. Se trata de técnicas conformadas por una subestructura de pies derechos de madera, dispuestos muy próximos entre sí, en los que se entrelazan varas flexibles, vergas, como las de fresno o sauce. Las fibras de esta pared pueden ser recubiertas con un revestimiento grueso de torta de barro, que da rigidez al cerramiento, protege la materia orgánica e incrementa la estanqueidad del espacio interior. También es posible encontrar zarzos sin revestimiento en espacios que, como los graneros, necesitan una buena ventilación.

Los mimbres son una variante de muro tejido en la que los espacios entre los soportes de madera son cerrados por mantas tejidas con una urdimbre de fibras o lizas (Fig. 9b). Gracias a su ligereza, en este tipo de muros la separación entre los pies derechos puede alcanzar distancias considerablemente mayores que las habituales en los muros tejidos y entramados, superando en ocasiones los 2 metros. Con el objetivo de aumentar la rigidez y la resistencia de estos tabiques, es habitual recubrirlos con un revoco de tierra o yeso.

En ocasiones, las fibras vegetales que forman las pantallas verticales no se encuentran entrelazadas entre los pies derechos, sino encajadas o ceñidas entre ellos. Tal es el caso de los cañizos (Fig. 9c), similares a las variedades latinoamericanas de quincha o bahareque. Estos sistemas se basan en la disposición apretada de cañas, horizontales y paralelas, para formar una pantalla que cierre el espacio entre dos postes de madera. Una vez se han encajado las cañas entre los postes, éstas se suelen recubrir con un revoco de tierra o yeso. Con frecuencia, este tipo de muros tejidos se emplea como cerramiento no portante en construcciones sustentadas por muros o pilares de piedra o de yeso y mampuestos. En estos casos, los pies derechos de la urdimbre sólo cuentan con un papel autoportante y pueden estar realizados con cañas. 
Los tabiques de tablas, o encamonados, se basan en la formación de una pantalla de tablas, de 10 a $20 \mathrm{~cm}$ de anchura y 3-4 cm de espesor, que se clava a la imprenta del muro (32). Sobre esta pantalla se fijan listones de madera o haces de fibras que se cubren con un revoco grueso de tierra y paja o tierra y cal (Fig. 9d). Estos elementos tienen una presencia remarcable en algunas zonas de Galicia, donde reciben el nombre de tabiques de pallabarro (25).

La pared de listón, o emparrillado, es una técnica similar al tabique encamonado, posiblemente más antigua, en la que los listones horizontales se fijan directamente a los montantes de la subestructura de madera y no se forma la pantalla de tablas. Los espacios existentes entre los listones se colmatan con una mezcla de barro y paja que llega a cubrir toda la superficie del muro tejido (14).

Los emparrillados dobles, también conocidos como tabiques de corres o tabiques de barrotes (32), son una variante de la pared de listón en la que las latas son clavadas por ambos lados de los montantes para formar una doble pantalla (Fig. 9e). Para su ejecución, se suelen clavar primero los listones de madera por la cara exterior de los pies derechos. Estas latas, que suelen tener un espesor de aproximadamente $1 \mathrm{~cm}$ y una anchura de entre 3 y $5 \mathrm{~cm}$, se colocan separadas entre 6 y $11 \mathrm{~cm}$ para formar un enrejado. A continuación, se rellena el espesor del muro con mampuestos pequeños, barro, ladrillos de desecho, cascotes o yesones trabados con yeso. El vertido se realiza asegurándose que la masa rebosa por el enrejado, antes de clavar la segunda pantalla de listones por la cara interior de los postes. Una vez completado el segundo enrejado, se vierte yeso claro o barro para trabarlo con el relleno. Cuando la fábrica ha fraguado, se aplica en ambos paramentos un revestimiento basto, sobre el que se extenderán después los revoques (6) (15). Además de estas mezclas de tierra arcillosa o yeso y cascotes, durante el desarrollo de esta investigación también ha sido posible registrar el empleo de mezclas de tierra, yeso y restos agrícolas (como mazorcas desgranadas) para la colmatación del espacio entre ambas parrillas (Fig. 9f).

\subsubsection{Revestimientos}

Con frecuencia, los muros mixtos de tierra y madera han sido revestidos para incrementar su durabilidad y dotarles de un acabado. En algunas técnicas, como los zarzos y los mimbres, el revoco es un elemento fundamental cuya función trasciende la mera protección y sin el cual no pueden desempeñar correctamente su papel. En estos casos, el revestimiento de tierra se suele recubrir a su vez con un encalado que prolonga su vida útil y que evita la proliferación de xilófagos. Por el contrario, diversas variedades de rellenos monolíticos, como el tapialete, suelen formar por sí mismos una superficie homogénea y resistente que hace innecesaria la aplicación de un acabado.

En las técnicas constructivas en las que el revestimiento mantiene su función habitual, la solución más frecuente es el trullado o revoco de barro y fibras. Estos revestimientos se realizan empleando una mezcla de composición similar a la de los adobes, pero que incorpora fibras de menor dimensión, como pelo de animal o paja muy picada. Cuando había cal disponible, sobre este revoco era común disponer un encalado que fijara las partículas, higienizara la construcción y la dotara de su aspecto final. En otras ocasiones, el revestimiento podía ser realizado empleando directamente materiales conglomerantes como enlucidos de yeso o revocos de mortero de cal (Fig. 10a). Otras variantes de revestimiento que se pueden encontrar asociadas a los muros mixtos son los revestimientos de tejas (Fig. 10b), los revestimientos de lajas de pizarra, los revestimientos de paja y los revestimientos de tablas (Fig. 10c), estos últimos producto de la vinculación de este sistema constructivo con entornos urbanos situados en zonas con abundante presencia de madera.

\section{DISCUSIÓN}

Bajo la denominación de muros mixtos de tierra y madera es posible agrupar un amplio abanico de soluciones constructivas que van desde los entramados con rellenos de albañilería

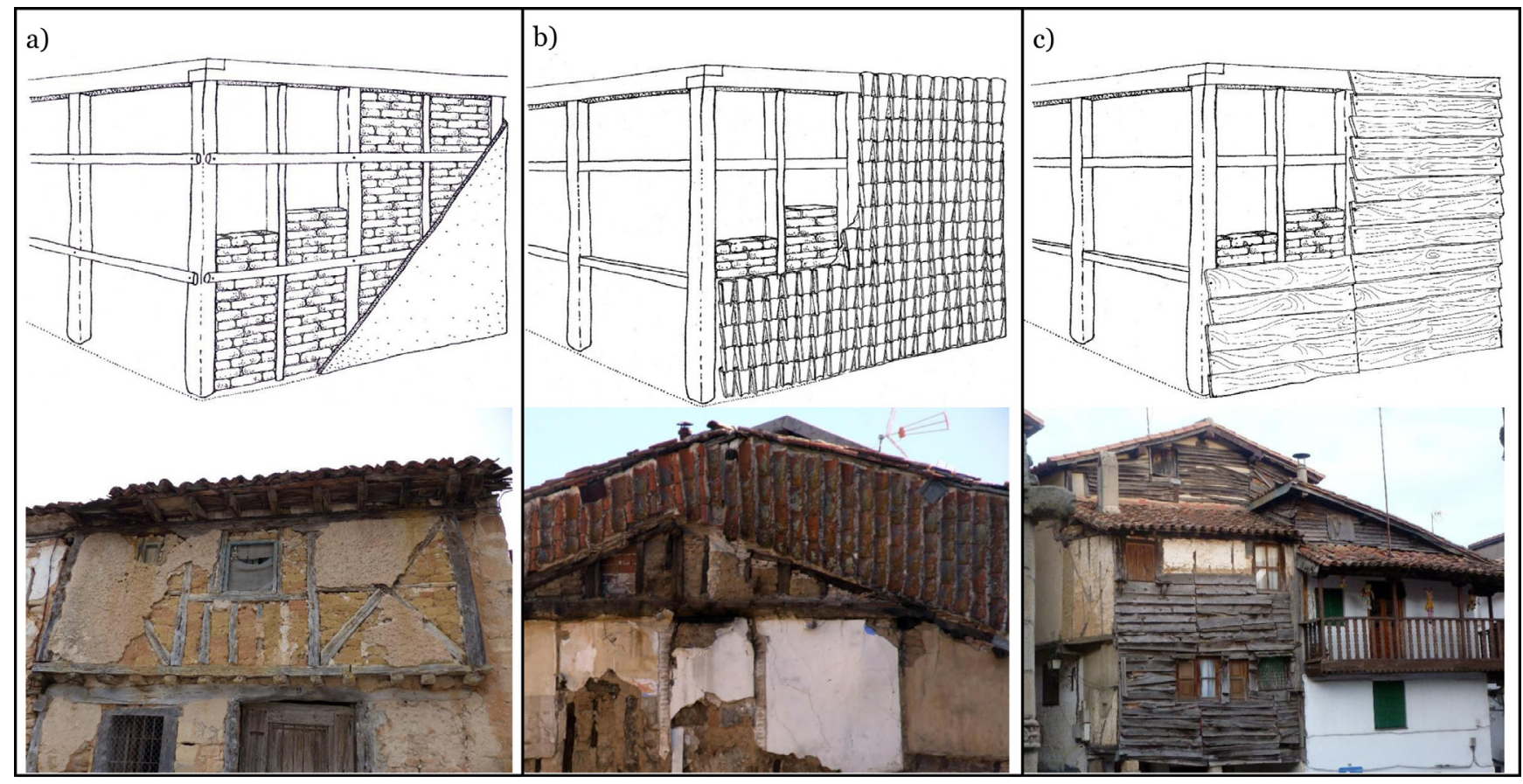

Figura 10. Revestimientos (Dibujos: SOStierra. Fotografías: F. Vegas y C. Mileto). 
(entramado con relleno de adobe horizontal aparejado, entramado con relleno de adobe en espiga, etc.), hasta los entramados con rellenos monolíticos (entramado relleno con pared de pellas, entramado relleno con tapialete, etc.) y los muros tejidos (zarzo, mimbre, verganazo, etc.).

Al observar los resultados arrojados por el análisis constructivo de la muestra, la colmatación de los cuarteles con rellenos ha demostrado ser mucho más frecuente que el uso de elementos tejidos. Mientras que un $89,9 \%$ de los casos estudiados se corresponden con este tipo de soluciones, sólo un 10,10\% de los edificios analizados están ejecutados con muros tejidos. Si se presta atención a los tipos de relleno empleados en los entramados, un 83'7\% de los casos registrados aparecen colmatados con fábricas de piezas de tierra, mientras que sólo un 16’3\% están resueltos con rellenos monolíticos. En la mayor parte de los casos, los rellenos de piezas están constituidos por adobes asentados en horizontal, bien aparejados o bien apilados. Los entramados rellenos con adobes dispuestos en diagonal o en espina de pez destacan más por su interés específico que por la frecuencia con la que han sido encontrados.

Los diseños empleados para resolver la subestructura de madera de entramados y muros tejidos resultan muy variados y aparecen de una forma más equilibrada que los materiales que los rellenan. Las imprentas simples, es decir, aquellas que cuentan únicamente con pies derechos de madera entre la solera y la carrera, suponen un 30'8\% de los casos observados. Por su parte, un $37^{\prime} 4 \%$ de los edificios estudiados incluyen puentes horizontales a mitad de muro y hasta un 49'1\% están reforza- dos con tornapuntas o cruces de San Andrés. Las diferentes formas en que se combinan estos tres tipos de elementos dan lugar a una gran riqueza creativa y estructural que merece el desarrollo de investigaciones específicas.

\section{CONCLUSIONES}

A pesar de partir de una lógica constructiva similar, este estudio ha demostrado la enorme riqueza y variedad de soluciones tradicionales de construcción que emplean elementos de tierra y madera. La metodología seguida en esta investigación es extrapolable para el estudio de los muros mixtos de tierra y madera en otros contextos diferentes del español (33). Los materiales disponibles en el entorno inmediato, la experiencia acumulada durante generaciones y el sentido de la oportunidad inherente a la arquitectura tradicional han dado lugar a un amplio rango de soluciones que se adaptan de forma óptima a las características de cada región, dando lugar a edificios confortables y de bajo impacto ambiental, económico y paisajístico de la que es posible extraer valiosas lecciones para el desarrollo de una arquitectura contemporánea más sostenible. Por último, el desarrollo de estudios como el presentado en este artículo resulta fundamental para difundir la percepción de esta arquitectura como un patrimonio global valioso que es necesario conservar y para proporcionar una base de conocimiento que permita el desarrollo de intervenciones compatibles con la naturaleza de estos edificios. El conocimiento detallado de los materiales y las técnicas de la construcción tradicional fomenta procesos de valorización cultural que superan ampliamente los límites locales y contribuyen a la creación de una conciencia patrimonial global.

\section{NOTA}

Este trabajo se enmarca dentro del proyecto de investigación "La restauración y rehabilitación de la arquitectura tradicional de tierra en la Península Ibérica. Líneas guía y herramientas para una intervención sostenible”, financiado por el Ministerio de Economía y Competitividad (BIA2014-55924-R), y de la tesis doctoral “Arquitectura tradicional de tierra en España. Caracterización constructiva, fenómenos de degradación y dinámicas de intervención” (27).

\section{REFERENCIAS / REFERENCES}

(1) Mileto, C., Vegas, F., Villacampa, L., García-Soriano, L. \& Gómez-Patrocinio, F. J. (2017). Vernacular earthen architecture in the Iberian Peninsula - First phase of taxonomy and geographical distribution. En Proceedings for the Ninth International Earthbuilding Conference (pp. 102-107). La Madera, New Mexico: Adobe in Action.

(2) López Martínez, F. J. (1999). Tapias y tapiales. Loggia. Arquitectura \& Restauración, 8, 74-89.

(3) Houben, H. \& Guillaud, H. (1984). Earth Construction I. Grenoble: Craterre.

(4) Vegas, F., Mileto, C., Cristini, V. \& García-Soriano, L. (2014). La tapia en la Península Ibérica. En F. Vegas \& C. Mileto (Eds.), La restauración de la tapia en la Península Ibérica. Criterios, técnicas, resultados y perspectivas (pp. 32-51). Lisboa: Argumentum Ediçóes. Valencia: TC Cuadernos.

(5) Gómez-Patrocinio, F. J., Vegas, F., Mileto, C. \& García-Soriano, L. (2019) Techniques and characteristics of traditional earthen masonry walls. The case of Spain. International Journal of Architectural Heritage. https://doi.org/10.1080/1 5583058.2018 .1563238

(6) Barré, L. A. (1899). Carpintería de armar. En Pequeña enciclopedia práctica de construcción. Madrid: Librería Editorial de Bailly-Bailliere e Hijos.

(7) Copani, P. (noviembre, 2007). Timber-frame buildings in Scandinavia: High deformation prevent the system from collapse. Trabajo presentado en From material to structure - Material behaviour and failures of timber structures. ICOMOS IWC - XVI International Symposium, Florencia, Venecia y Vicenza.

(8) Ceccotti, A., Faccio, P., Nart, M., Sandhaas, C. \& Simeone, P. (septiembre, 2006). Seismic behaviour of historic timber-frame buildings in the Italian Dolomites. Trabajo presentado en ICOMOS IWC - XV International Symposium, Estambul y Rize.

(9) González-Redondo, E. (2014). De la construcción entramada a la introducción de las estructuras metálicas en Madrid. En Informes de la Construcción, 66:534 (pp. 1-11). https://doi.org/10.3989/ic.12.100

(10) Vargas, J., Iwaki, C. \& Rubiños, A. (2012). Seismic resistance in the core of Caral, Peru. En Proceedings of Terra 2012 / $12^{\text {th }}$ SIACOT (pp. 239-243). Lisboa: Argumentum Ediçóes. 
(11) De Hoz, J., Maldonado, L. \& Vela, F. (2003). Diccionario de construcción tradicional con tierra. San Sebastián: Ed. Nerea.

(12) Marcos y Bausá, R. (1879). Manual del Albañil. Madrid: Dirección y Administración.

(13) Gil Crespo, I. (2014). Geographical cataloguing of earthen architecture in Soria, Spain. En Vernacular heritage and earthen architecture. Contributions for sustainable development (pp. 123-128). Londres: Taylor \& Francis.

(14) Font, J., Vegas, F. \& Mileto, C. (2017). Técnicas y puesta en obra en España. En Proyecto Coremans. Criterios de intervención en la arquitectura de tierra (pp. 46-73). Madrid: Ministerio de Educación, Cultura y Deporte.

(15) Ger y Lóbez, F. (1898). Tratado de construcción civil. Badajoz: La Minerva Extremeña.

(16) Guillaud. H. (2012). Pisé: Evolution, innovations, resistances and future directions. En Rammed Earth Conservation (pp. 3-8). Londres: Taylor \& Francis.

(17) Joffroy, T., Guillaud, H. \& Salazar, C. (Eds.) (2016) The Lyon Declaration. En International Conference Terra Lyon 2016. XIIth World Congress on Earthen Architecture. Villefontaine: Éditions Craterre.

(18) Warren, J. (1993). Earth conservation: the conservation of brick and earth structures. A handbook. Columbo: Sri Lanka National Committee of ICOMOS.

(19) Roldán, F. P. (1983). Palomares de barro en Tierra de Campos. Valladolid: Caja de Ahorros de Valladolid.

(20) Alonso, J. L. \& Cid, S. (1994). La arquitectura del barro. Valladolid: Junta de Castilla y León.

(21) Vegas, F. \& Mileto, C. (Eds.) (2014). La restauración de la tapia en la Península Ibérica. Criterios, técnicas, resultados y perspectivas. Lisboa: Argumentum Ediçóes. Valencia: TC Cuadernos.

(22) Canivell, J. (2011). Metodología de diagnóstico y caracterización de fábricas históricas de tapia (Tesis doctoral). Universidad de Sevilla, Sevilla, España.

(23) Espinosa, P. C. (1859). Manual de construcciones de albañilería. Madrid: Imprenta de D. Severiano Baz.

(24) Font, J. (2012) Constructive systeme in the Spanish North-Western area. En Rammed Earth Conservation (pp. 511516). Londres: Taylor \& Francis.

(25) Fernández Palicio, A. (2015). Earthen construction in Alto Arnoia river valley, Galicia. En Earthen Architecture: Past, Present \& Future (pp. 139-144). Londres: Taylor \& Francis.

(26) Nuere, E. (2000). La carpintería de armar española. Madrid: Munillalería.

(27) Gómez-Patrocinio, F. J. (2018) Arquitectura tradicional de tierra en España. Caracterización constructiva, fenómenos de degradación y dinámicas de intervención. Tesis doctoral. Valencia: Universitat Politècnica de València.

(28) Fuentes, J.M. \& Cañas, I. (2003) Estudio y caracterización de la arquitectura rural. Obtención, tratamiento y manejo de la información sobre las construcciones. Informes de la Construcción, 55:487 (pp. 13-21). doi:10.3989/ic.2003.v55. i487.544

(29) Porto, S. \& Cascone, G. (2013) Método de construcción en base a la caracterización orientado a fomentar el conocimiento de las características arquitecturales externas de edificaciones rurales tradicionales. Informes de la Construcción, 65:532 (pp. 481-496). doi: 10.3989/ic.12.086

(30) López-Roldán, P. \& Fachelli, S. (2015). El diseño de la muestra. En Metodología de la investigación social cuantitativa, (pp. 5-58). Barcelona: Universitat Autònoma de Barcelona.

(31) Vegas, F., Mileto. C., Gómez-Patrocinio, F. J. \& Pérez Vila, A. (2018). Constructive analysis of the rammed earth walls in the Petrés Castle (Valencia). En Vernacular and earthen architecture. Conservation and sustainability (pp. 499-504). Londres: Taylor \& Francis.

(32) Fernández Palicio, A. (2012). Earthen architecture in Galicia (Spain). Rammed earth in Terra de Lemos. En Rammed Earth Conservation (pp. 99-105). Londres: Taylor \& Francis.

(33) Correia, M., Dipasquale, L. \& Mecca, S. (2011). Terra Europae. Earthen Architecture in the European Union. Pisa: Edizioni ETS. Tervuren: Culture Lab. 